Pacific Journal of Mathematics

GENERALIZED FINAL RANK FOR ARBITRARY LIMIT 


\title{
GENERALIZED FINAL RANK FOR ARBITRARY LIMIT ORDINALS
}

\author{
Doyle O. Cutler and Paul F. Dubois
}

Let $G$ be a $p$-primary Abelian group. The final rank of $G$ can be obtained in two equivalent ways: either as $\inf _{n \in \omega}\left\{\boldsymbol{r}\left(p^{n} G\right)\right\}$ where $r\left(p^{n} G\right)$ is the rank of $p^{n} G$; or as $\sup \{r(G / B) \mid B$ is a basic subgroup of $G\}$. In fact it is known that there exists a basic subgroup of $G$ such that $r(G / B)$ is equal to the final rank of $G$. In this paper are displayed two appropriate generalizations of the above definitions of final rank, $r_{\alpha}(G)$ and $s_{\alpha}(G)$, where $\alpha$ is a limit ordinal. It is shown that the two cardinals $r_{\alpha}(G)$ and $s_{\alpha}(G)$ are indeed the same for any limit ordinal $\alpha$. In this context one can think of the usual final rank as " $\omega$-final rank".

The final rank of a $p$-primary Abelian group $G$ is $\inf _{n<\omega}\left\{r\left(p^{n} G\right)\right\}$ where $r\left(p^{n} G\right)$ means the rank of $p^{n} G$. The same cardinal number is obtained by taking $\sup _{B \in \Gamma} r(G / B)$ where $\Gamma$ is the set of all basic subgroups of $G$. In [1] we defined for limit ordinals $\alpha, s_{\alpha}(G)=\inf _{\beta<\alpha} r\left(p^{\beta} G\right)$ and $r_{\alpha}(G)=\sup _{H \in \Gamma} r(G / H)$ where $\Gamma$ is the set of all $p^{\alpha}$-pure subgroups $H$ of $G$ such that $G / H$ is divisible; it was shown that for accessible ordinals $\alpha$ that $r_{\alpha}(G)=s_{\alpha}(G)$. The proof given there strongly depended on the accessibility of $\alpha$. In this paper it is proved that $r_{\alpha}(G)=s_{\alpha}(G)$ for any limit ordinal $\alpha$, at the cost of a considerably more difficult argument.

Throughout we consider a reduced $p$-primary Abelian group $G$. We consider cardinal and ordinal numbers in the sense of von Neumann; that is, an ordinal number is a set, namely, the set of all smaller ordinals. Cardinal numbers are ordinal numbers that are not equivalent to any smaller ordinal. The cardinal number of a set $\Gamma$ is denoted by $|\Gamma|$. The symbol $\omega$ denotes the first infinite ordinal. In general the notation and terminology is that of [2] or [3].

1. The lemmas. Let $\tau$ be a limit ordinal. We define the final $\tau$-rank of $G$ in two ways, which we will then show are equivalent. Ordinary final rank as defined in [2] corresponds to final $\omega$-rank.

\section{DEFINITION.}

(1) $s_{\tau}(G)=\inf _{\beta<\tau} r\left(p^{\beta} G[p]\right)$.

(2) $r_{\tau}(G)=\sup \{r(G / H): H \subseteq G, G / H$ is divisible, and $0 \rightarrow H \rightarrow$ $G \rightarrow G / H \rightarrow 0$ represents an element of $\left.p^{\tau} \operatorname{Ext}(G / H, H)\right\}$.

In [1] it is shown that $r_{\tau}(G) \leqq s_{\tau}(G)$. To show the converse we 
will construct a $p^{\tau}$-pure subgroup $H$ of $G$ with $G / H$ divisible and $r(G / H)=s_{\tau}(G)$.

We prove the following lemma to simplify the problem and to illustrate some techniques of construction which we will sometimes use later in the paper without explicit proofs.

LEMMA 0. (a) $r_{\tau}(G)=r_{\tau}\left(G_{\tau}\right)+r\left(p^{\tau} G\right)$ for any $p^{\tau} G$-high subgroup $G_{\tau}$.

(b) $s_{\tau}(G)=s_{\tau}\left(G_{\tau}\right)+r\left(p^{\tau} G\right)$ for any $p^{\tau} G$-high subgroup $G_{\tau}$.

(c) $r_{\tau}(G) \geqq s_{\tau}(G)$ holds for all $G$ if it holds for all $G$ satisfying $p^{\tau} G=0$.

Proof. (a) and (b) together show (c). A $p^{\tau} G$-high subgroup $G_{\tau}$ satisfies $G[p]=G_{\tau}[p] \oplus\left(p^{\tau} G\right)[p],([4])$ and hence is $p^{\tau+1}$-pure (Th. 2.9 of [5]). It is easy to see that for $\alpha<\tau$,

$$
\left(p^{\alpha} G\right)[p]=\left(p^{\alpha} G_{\tau}\right)[p] \oplus\left(p^{\tau} G\right)[p]
$$

and (b) follows.

To prove (a), suppose $H$ is a $p^{\tau}$-pure subgroup of $G_{\tau}$ with $G_{\tau} / H$ divisible. Then $H$ is $p^{\tau}$-pure in $G$ and $G / H \cong\left(G / G_{\tau}\right) /\left(G_{\tau} / H\right)$ is divisible since $G / G_{\tau}$ is divisible. For $H$ a pure subgroup of $G$,

$$
r(G / H)=r((G / H)[p])=r(G[p] / H[p]) .
$$

Hence in this case

$$
r(G / H)=r\left(G_{\tau}[p] \oplus\left(p^{\tau} G\right)[p] / H[p]\right)=r\left(G_{\tau} / H\right)+r\left(p^{\tau} G[p]\right) .
$$

Hence $r_{\tau}(G) \geqq r_{\tau}\left(G_{\tau}\right)+r\left(p^{\tau} G\right)$.

Now let $H$ be a $p^{\tau}$-pure subgroup of $G$ with $G / H$ divisible. Let $H[p]=S \oplus\left(p^{\tau} G \cap H\right)[p]$. Let $K$ be a $p^{\tau} G$-high subgroup containing $S$, and let $\pi: G \rightarrow G / p^{\tau} G$ be the natural map. Then $(\pi(K))[p]=\pi(K[p])=$ $\pi\left(G_{\tau}[p]\right)$. Choose $S^{\prime} \subseteq G_{\tau}[p]$ such that $\pi\left(S^{\prime}\right)=\pi(S)$. We will then have that $r\left(G_{\tau}[p] / S^{\prime}\right)=r\left(\pi\left(G_{\tau}\right) / \pi(S)\right)=r(K[p] / S)$. Note that $\left\{S^{\prime},\left(p^{\tau} G\right)[p]\right\}=$ $\left\{S,\left(p^{\tau} G\right)[p]\right\}$ and so the $p^{\tau}$-purity of $H$ and the divisibility of $G / H$ yield, for every $\alpha<\tau$,

$$
\begin{aligned}
\left\{p^{\alpha} G_{\tau}[p], S^{\prime}\right\} & =\left\{\left(p^{\alpha} G \cap G_{\tau}\right)[p], S^{\prime}\right\} \\
& =\left\{\left\{p^{\alpha} G[p], S^{\prime}\right\} \cap G_{\tau}[p]\right\} \\
& =\left\{\left\{p^{\alpha} G[p], S\right\} \cap G_{\tau}[p]\right\} \\
& =\left\{G[p] \cap G_{\tau}[p]\right\}=G_{\tau}[p] .
\end{aligned}
$$

We let $L$ be such that $G_{\tau}[p]=L \oplus S^{\prime}$ and let $M$ be $L$-high containing $S^{\prime}$. Then $M[p]=S^{\prime}, M$ is neat in $G_{\tau}[p]$ and by Th. 2.9 of [5], $M$ is $p^{\tau}$-pure in $G_{\tau}$. Then 


$$
\begin{aligned}
r(G / H) & =r\left(\left(K[p] \oplus\left(p^{\tau} G\right)[p]\right) /\left(S \oplus\left(p^{\tau} G \cap H\right)[p]\right)\right) \\
& =r(K[p] / S)+r\left(p^{\tau} G[p] /\left(p^{\tau} G \cap H\right)[p]\right) \\
& \leqq r\left(G_{\tau}[p] / S^{\prime}\right)+r\left(p^{\tau} G[p]\right) \\
& =r\left(G_{\tau} / M\right)+r\left(p^{\tau} G\right) \\
& \leqq r_{\tau}\left(G_{\tau}\right)+r\left(p^{\tau} G\right)
\end{aligned}
$$

and (a) is proved.

Hence we consider only groups $G$ with $p^{\tau} G=0$. We will need the following four technical lemmas.

Lemma 1. Let $G$ be a p-primary Abelian group of length $\tau$, a limit ordinal. Let $S \subseteq G[p]$ be such that $S \cap\left(p^{r} G\right)[p] \neq 0$ for all $\gamma<\tau$. Then there exists $S^{\prime} \leqq S$ such that $r\left(S / S^{\prime}\right) \geqq 1$ and $\left\{S^{\prime},\left(p^{\gamma} G\right)[p]\right\}=$ $\left\{S,\left(p^{\gamma} G\right)[p]\right\}$ for all $\gamma<\tau$.

Proof. Let $a \in S(a \neq 0)$. We define a family $\left\{R_{j}\right\}_{j<:}$ inductively as follows:

Write $S=L_{1} \oplus p G \cap S$. If $a \notin L_{1}$, let $R_{1}=L_{1}$. If $a \in L_{1}$, let $\left\{y_{\alpha}\right\}_{\alpha \in \Gamma}$ be a basis for $L_{1}$. Then $a=\sum_{\alpha \in \Gamma} a_{\alpha} y_{\alpha}$ where $0 \leqq a_{\alpha}<p$ and $a_{\alpha}=0$ for all but finitely many $\alpha$. Choose $\alpha_{0} \in \Gamma$ so that $a_{\alpha_{0}} \neq 0$. Let $R_{1}=\sum_{\alpha \in \Gamma-\left\{\alpha_{0}\right\}}\left\langle y_{\alpha}\right\rangle \oplus\left\langle y_{\alpha_{0}}-b\right\rangle$ where $b \in p G \cap S(b \neq 0)$. Then $S=$ $R_{1} \oplus p G \cap S$ and $a \notin R_{1}$. Inductively, suppose $\left\{R_{i}\right\}_{i<r}$ has been defined such that $\sum_{i \leqq k<r} R_{i} \oplus p^{k} G \cap S=S$ for each $k<\gamma$ and $a \notin \sum_{i<r} R_{i}$. If $\gamma-1$ exists we have $\sum_{i<r} R_{i} \oplus p^{p^{-1}} G \cap S=S$. We choose $L_{\gamma}$ so that $L_{r} \oplus p^{r} G \cap S=p^{-1} G \cap S$. If $a \notin \sum_{i<r} R_{i} \oplus L_{\gamma}$ we let $R_{\gamma}=L_{i}$. Otherwise, let $\left\{y_{\lambda}\right\}_{\lambda \in \Gamma}$ be a basis of $L_{\gamma}$. Then $a=x+\sum_{\lambda \in \Gamma} a_{\lambda} y_{\lambda}\left(0 \leqq \alpha_{\lambda}<p\right.$, $\left.x \in \sum_{i<r} R_{i}\right)$. By the induction hypothesis not all $a_{\lambda}$ are zero. Let $\lambda_{0} \in \Gamma$ such that $a_{\lambda_{0}} \neq 0$, and let $R_{\gamma}=\sum_{\lambda \in \Gamma-\left\{\lambda_{0}\right\}}\left\langle y_{\lambda}\right\rangle \oplus\left\langle y_{\lambda_{0}}-b\right\rangle\left(b \in p^{r} G \cap\right.$ $S, b \neq 0)$. It follows that $a \notin \sum_{i<\gamma+1} R_{i}$ and $\sum_{i \leqq k \leqq \gamma} R_{i} \oplus p^{k} G \cap S=S$.

If $\gamma$ is a limit ordinal, note that $\sum_{i<r} R_{i} \cap p^{\gamma} G=0$. Choose $L_{\gamma}$ such that $\sum_{i<r} R_{i} \oplus L_{\gamma} \oplus p^{\gamma} G \cap S=S$. Either $a \notin \sum_{i<r} R_{i} \oplus L_{\gamma}$ in which case we let $R_{\gamma}=L_{\gamma}$, or $a \in \sum_{i<r} R_{i} \oplus L_{\gamma}$ and we modify $L_{\gamma}$ as above to get $R_{r}$.

By transfinite induction, we obtain a family $\left\{R_{i}\right\}_{i<\tau}$ such that $\sum_{i \leqq k} R_{i} \oplus p^{k} G \cap S=S$ for all $k<\tau$ and $a \notin \sum_{i<=} R_{i}$. Let $S^{\prime}=\sum_{i<\tau} R_{i}$ and the conditions of the lemma are satisfied.

The general idea of the above proof for $S$ summable was communicated to the authors by Paul Hill.

Lemma 2. Let $G$ be a p-primary Abelian group of length $\tau$ a limit ordinal. Let $\left\{R_{j}\right\}_{j<\eta}, \eta$ a limit ordinal, be a collection of subsocles of $G$ satisfying the following conditions: 
(1) $\sum_{j<\eta} R_{j}$ is direct,

(2) $r\left(R_{j}\right)=\aleph$ is fixed, and

(3) For each $\lambda<\tau$, there exists $j<\eta$ such that $0 \neq R_{j} \subseteq p^{2} G[p]$. Then there exists $S \subseteq \sum_{j<\eta} R_{j}$ such that

(a) For each $\lambda<\tau,\left\{S, p^{\lambda} G[p]\right\}=\left\{\sum_{j<\eta} R_{j}, p^{\lambda} G[p]\right\}$, and

(b) $r\left(\left(\sum_{j<\eta} R_{j}\right) / S\right) \geqq \aleph$.

Proof. For each $j<\eta$, let $\left\{x_{j, \alpha}\right\}_{\alpha \in \Gamma}(|\Gamma|=\aleph)$ be a basis of $R_{j}$. Let $S_{\alpha}=\sum_{j<\eta}\left\langle x_{j \alpha}\right\rangle$. Note that $\sum_{\alpha \in \Gamma} S_{\alpha}$ is direct and $\sum_{\alpha \in \Gamma} S_{\alpha}=\sum_{\alpha<\eta} R_{j}$. Let $\lambda<\tau$. Then $S_{\alpha} \cap p^{\lambda} G[p] \neq 0$ by hypothesis (3). Hence by Lemma 1 , there exists, for each $\alpha \in \Gamma, T_{\alpha} \subseteq S_{\alpha}$ such that

$$
\left\{S_{\alpha}, p^{\lambda} G[p]\right\}=\left\{T_{\alpha}, p^{\lambda} G[p]\right\}
$$

for all $\lambda<\tau$ and $r\left(S_{\alpha} / T_{\alpha}\right) \geqq 1$. Let $S=\sum_{\alpha \in \Gamma} T_{\alpha}$. Then $\left\{S, p^{\lambda} G[p]\right\}=$ $\left\{\sum S_{\alpha}, p^{\lambda} G[p]\right\}=\left\{\sum_{j<\eta} R_{j}, p^{\lambda} G[p]\right\}$ for all $\lambda<\tau$ and $r\left(\left(\sum_{j<\eta} R_{j}\right) / S\right)=$ $\sum_{\alpha \in \Gamma} r\left(S_{\alpha} / T_{\alpha}\right) \geqq \boldsymbol{K}$.

Lemma 3. Let $G$ be a p-primary Abelian group of length $\tau$ a limit ordinal. Let $\sigma$ be an infinite initial ordinal such that $\sigma \leqq \tau$. Let $\left\{R_{j}\right\}_{j<\sigma}$ be a collection of subsocles of $G$ satisfying:

(1) $\sum_{j<\sigma} R_{j}$ is direct.

(2) For each $\lambda<\tau$ there exists $j<\sigma$ such that for all $i \geqq j$, $R_{i} \subseteq p^{\lambda} G[p]$.

(3) $\left|\left\{j \mid R_{j} \neq 0\right\}\right|=\sigma$.

Then there exists $S \subseteq \sum_{j<\sigma} R_{j}$ such that

(a) $\left\{S, p^{\lambda} G[p]\right\}=\left\{\sum_{j<\sigma} R_{j}, p^{\lambda} G[p]\right\}$ for all $\lambda<\tau$, and

(b) $\left|\left(\sum_{j<\sigma} R_{j}\right) / S\right| \geqq \sigma$.

Proof. Let $x_{j} \in R_{j}\left(x_{j} \neq 0\right)$ for each $j \in\left\{j \mid R_{j} \neq 0\right\}=\Gamma$. Then we may write $\Gamma$ as the disjoint union $\Gamma=\mathrm{U}_{i<o} \Gamma_{i}$ such that $\left|\Gamma_{i}\right|=\sigma$ for each $i<\sigma$. Since $\sigma$ is an initial ordinal, $\Gamma_{i} \nsubseteq \beta$ for any $\beta<\sigma$. Hence $\sum_{j \in \Gamma_{i}}\left\langle x_{j}\right\rangle$ satisfies the conditions of Lemma 1. Hence there exists a subgroup $S_{i} \subseteq \sum_{j \in \Gamma_{i}}\left\langle x_{j}\right\rangle$ such that $\left\{S_{i}, p^{\lambda} G[p]\right\}=\left\{\sum_{j \in \Gamma_{i}}\left\langle x_{j}\right\rangle\right.$, $\left.p^{\lambda} G[p]\right\}$ for all $\lambda<\tau$, and $r\left(\left(\sum_{j \in \Gamma_{i}}\left\langle x_{j}\right\rangle\right) / S_{i}\right) \geqq 1$. Let $Q$ be such that $\sum_{i<\sigma} \sum_{r_{i}}\left\langle x_{j}\right\rangle \oplus Q=\sum_{j<\sigma} R_{j}$, and define $S=\sum_{i<\sigma} S_{i} \oplus Q$. Then $S$ satisfies the desired conditions.

Lemma 4. Let $G$ be a p-primary Abelian group of length $\tau$ a limit ordinal. Let $\left\{R_{j}\right\}_{j \in \sigma}(\sigma$ a limit ordinal, $\sigma \leqq \tau)$ be a collection of subsocles of $G$ satisfying:

(1) $\sum_{j<\sigma} R_{j}$ is direct;

(2) For each $\lambda<\tau$, there exists $j<\sigma$ such that for all $j<i<\sigma$, $R_{i} \subseteq p^{\lambda} G[p]$; and

(3) For all $i<j<\sigma, r\left(R_{j}\right) \geqq r\left(R_{i}\right) \geqq|\sigma|$. 
Then there exists a subgroup $S \subseteq \sum_{j<\sigma} R_{j}$ satisfying:

(a) $\left\{S, p^{\lambda} G[p]\right\}=\left\{\sum_{j<\sigma} R_{j}, p^{\lambda} G[p]\right\}$ for all $\lambda<\tau$.

(b) $\left|\left(\sum_{j<\sigma} R_{j}\right) / S\right|=\left|\sum_{j<\sigma} R_{j}\right|$.

Proof. Define $Q_{\beta}^{\alpha}$ for all $(\alpha, \beta) \in \sigma x \sigma$ as follows: Define $Q_{0}^{0}=R_{0}$ and $Q_{0}^{\alpha}=0$ for all $\alpha<\sigma, \alpha>0$. We induct on the lower index. Suppose $Q_{\beta}^{\alpha}$ has been defined for all $\beta<\gamma<\sigma$ satisfying:

(1) For all $\alpha \leqq \beta<\gamma, r\left(Q_{\beta}^{\alpha}\right)=r\left(Q_{\alpha}^{\alpha}\right)$;

(2) $Q_{\beta}^{\alpha}=0$ if $\beta<\alpha<\sigma$;

(3) For $\beta<\sigma, r\left(Q_{\beta}^{\beta}\right) \neq 0$ if and only if $r\left(R_{\beta}\right)>r\left(R_{\alpha}\right)$ for all $\alpha<\beta$; and

(4) $R_{\beta}=\sum_{\alpha \in \sigma} Q_{\beta}^{\alpha}$.

Suppose $\gamma-1$ exists. If $r\left(R_{\gamma}\right)=r\left(R_{\gamma-1}\right)$, let $\varphi: R_{\gamma-1} \rightarrow R_{\gamma}$ be an isomorphism and define $Q_{\gamma}^{\alpha}=\varphi\left(Q_{\gamma-1}^{\alpha}\right)$ for all $\alpha \in \sigma$. If $r\left(R_{\gamma-1}\right)<r\left(R_{\gamma}\right)$ we first write $R_{r}=S \oplus R$ where $R \cong R_{\gamma-1}$ (under an isomorphism $\varphi$ ). Let $Q_{\gamma}^{\alpha}=\varphi\left(Q_{\gamma-1}^{\alpha}\right)$ for $\alpha<\gamma, Q_{\gamma}^{\gamma}=S$, and $Q_{r}^{\alpha}=0$ for $\alpha>\gamma$.

Suppose $\gamma$ is a limit ordinal. If $r\left(R_{\gamma}\right)=r\left(R_{\beta}\right)$ for some $\beta<\gamma$, then $R_{\gamma} \cong R_{\alpha}$ for all $\beta \leqq \alpha<\gamma$. Let $\varphi$ be an isomorphism from $R_{\beta}$ onto $R_{\gamma}$ and let $Q_{r}^{\alpha}=\varphi\left(Q_{\beta}^{\alpha}\right)$ for all $\alpha<\sigma$.

If for some $\beta<\gamma, r\left(R_{\gamma}\right)>r\left(R_{\beta}\right) \geqq r\left(R_{\alpha}\right)$ for all $\alpha<\gamma$ we write $R_{r}=R \oplus S$ where $R \cong R_{\beta}$ and proceed as in the case of the nonlimit ordinal.

Finally suppose $r\left(R_{\gamma}\right)>r\left(R_{\beta}\right)$ for all $\beta<\gamma$ and that there does not exist $\delta<\gamma$ such that $r\left(R_{\delta}\right) \geqq r\left(R_{\beta}\right)$ for all $\beta<\gamma$. Let $\pi=\sum_{\beta<r} r\left(R_{\beta}\right)$. Since $r\left(R_{\gamma}\right)>r\left(R_{\beta}\right) \geqq|\sigma|, \beta<\gamma$, we have $\pi \leqq r\left(R_{r}\right)$ and both of these cardinals are infinite. We may write $R_{r}$ as $S \oplus R$ where $r(S)=\pi$. Divide a basis of $S$ into two sets, $\left\{y_{\lambda}\right\}_{\lambda \in \pi}$ and $\left\{z_{\lambda}\right\}_{\lambda \in \pi}$. Let $Q_{r}^{r}=R \oplus$ $\left\langle\left\{z_{\lambda}\right\}_{\lambda \in \pi}\right\rangle$, and noting that $\pi=\sum_{\alpha<r} r\left(Q_{\alpha}^{\alpha}\right)$, write $\pi$ as the disjoint union $\pi=\mathbf{U}_{\alpha<r} \pi_{\alpha}$ such that $\left|\pi_{\alpha}\right|=r\left(Q_{\alpha}^{\alpha}\right)$.

Let $Q_{r}^{\alpha}=\left\langle\left\{y_{\lambda} \mid \lambda \in \pi_{\alpha}\right\}\right\rangle$, and we complete the induction. Note that by the construction that if $R_{r}$ is the first to have rank $\rho$, then $r\left(Q_{r}^{r}\right)=\rho$.

Let $\Lambda=\left\{r\left(R_{j}\right) \mid j<\sigma\right\}$. For each $\rho \in \Lambda$ let $j_{\rho}$ be the least element of $\sigma$ such that $r\left(R_{j_{\rho}}\right)=\rho$. Then $Q_{j_{\rho}^{j}}^{j_{\rho}} \neq 0$ by construction. For each $\rho \in \Lambda$ consider the collection $\left\{Q_{\alpha}^{j}\right\}_{\alpha \in \Gamma_{\rho}}$ where $\Gamma_{\rho}=\left\{j \mid j_{\rho} \leqq j<\sigma\right\}$. Note that this collection satisfies the hypothesis of Lemma 2. Thus there exists a subgroup $S_{\rho} \leqq \sum_{\alpha \in \Gamma_{\rho}} Q_{\alpha}^{j}{ }^{\rho}$ such that $\left|\left(\sum_{\alpha \in \Gamma_{\rho}} Q_{\alpha^{\rho}}^{j}\right) / S_{\rho}\right| \geqq \rho$, and for each $\lambda<\tau,\left\{S_{\rho}, p^{\lambda} G[p]\right\}=\left\{\sum_{\alpha \in \Gamma_{\rho}} Q_{\alpha^{\prime}}^{j}, p^{\lambda} G[p]\right\}$. Note that

$$
\sum_{\rho \in A} \sum_{\alpha \in \Gamma_{\rho}^{\prime}} Q_{\alpha}^{j} \rho=\sum_{j<\sigma} R_{j}
$$

since each nonzero $Q_{\alpha}^{\alpha}$ is a $Q_{j \rho}^{j} \rho$ for some $\rho \in \Lambda$. Let $S=\sum_{\rho \in \Lambda} S_{\rho}$. Then

$$
\left|\left(\sum_{j<\sigma} R_{j}\right) / S\right|=\sum_{\rho \in A}\left|\left(\sum_{\alpha \in \Gamma} Q_{\alpha}^{j} \rho\right) / S_{\rho}\right| \geqq \sum_{\rho \in A} \rho=\sum_{j<\sigma} r\left(R_{j}\right)=\left|\sum_{j<\sigma} R_{j}\right| \cdot
$$


(Note that we use the last part of condition (3) for the second equality). Also for each $\lambda<\tau,\left\{S,\left(p^{\lambda} G\right)[p]\right\}=\left\{\sum_{j<\sigma} R_{j},\left(p^{\lambda} G\right)[p]\right\}$.

\section{The Theorem.}

THEOREM. Let $G$ be a reduced p-primary Abelian group. Then $r_{\tau}(G)=s_{\tau}(G)$.

Proof. As indicated in the introduction we may assume that the length of $G$ is $\tau$. Let $\lambda<\tau$ be such that $\left|p^{\lambda} G\right|=s_{\tau}(G)$. Then there exists an ordinal $\beta$ such that $\tau=\lambda+\beta$, and the length of $p^{\lambda} G$ is $\beta$. Now $r_{\tau}(G) \geqq r_{\beta}\left(p^{\lambda} G\right)$ (Use [5, Th. 2.9]) and $s_{\tau}(G)=s_{\beta}\left(p^{\lambda} G\right)$. Hence we need only show $r_{\beta}\left(p^{\lambda} G\right)=s_{\beta}\left(p^{\lambda} G\right)$. Thus we may consider only those groups $G$ with length $\tau$ and $r(G)=s_{\tau}(G)$.

Let $\Gamma$ be the set of all ordinals $\beta$ such that there exists a oneto-one order preserving map $f_{\beta}$ from $\beta$ into $\tau$ such that $\bigcup_{\alpha<\beta} f_{\beta}(\alpha)=\tau$. Let $\sigma$ be the least element of $\Gamma$ and $f=f_{\sigma}$. It follows easily from Theorem 13.4.4 of [6] that $\sigma$ is an initial ordinal.

Define a set of subgroups $\left\{P_{\alpha}\right\}_{\alpha<\sigma}$ of $G[p]$ as follows: Let $P_{0}$ be such that $G[p]=P_{0} \oplus\left(p^{f(0)} G\right)[p]$. Assuming that $P_{\alpha}$ has been defined for all $\alpha<\beta<\sigma$, define $P_{\beta}$ such that $G[p]=\sum_{\alpha<\beta} P_{\alpha} \oplus P_{\beta} \oplus\left(p^{f(\beta)} G\right)[p]$. This procedure is inspired by [3].

Choose $\lambda_{0}$ such that $\left|\sum_{\lambda_{0} \leqq i<\sigma} P_{i}\right|=\inf _{\lambda<o}\left|\sum_{\lambda \leqq i<\sigma} P_{i}\right| . \quad$ By the choice of $\sigma$, we have that $\left[\lambda_{0}, \sigma\right)=\sigma$. Hence we assume henceforth that $\left|\sum_{i<\sigma} P_{i}\right|=\inf _{\lambda<\sigma}\left|\sum_{\lambda \leqq i<\sigma} P_{i}\right|$. We may, in fact, assume each $P_{\alpha}, \alpha<\sigma$ is nonzero, again because $\sigma$ is regular (see [6]).

Let $Q$ be such that $\sum_{i<\sigma} P_{i} \oplus Q=\sum_{i<\sigma}$ (the original $P_{i}$ ). Then note that for each $\lambda<\tau,\left\{\sum_{i<\sigma} P_{i} \oplus Q,\left(p^{2} G\right)[p]\right\}=G[p]$. Let $M=$ $\sum_{i<\sigma} P_{i}$.

Case I. Suppose $|M|<s_{\tau}(G)$. Then [5, Th. 2.9] $M \oplus Q$ supports a $p^{\tau}$-pure subgroup $K$ of $G$ with $G / K$ divisible and $|G / K| \geqq s_{\tau}(G)$. So we assume $|M| \geqq s_{\tau}(G)$.

Case II $(A)$. Suppose $|M|=\sigma$. Then by Lemma 3 there exists a subsocle $S$ of $M$ such that $|M / S| \geqq \sigma \geqq s_{\tau}(G)$, and $\left\{S,\left(p^{\lambda} G\right)[p]\right\}=$ $\left\{M,\left(p^{2} G\right)[p]\right\}$ for all $\lambda<\tau$. Thus $S \oplus Q$ supports a $p^{\tau}$-pure subgroup $K$ of $G$ with $G / K$ divisible and $|G / K| \geqq s_{\tau}(G)$.

$(B)$. Suppose $|M|>\sigma$. Then construct a family of subsocles $\left\{R_{i}\right\}_{i \leqq \sigma}$ inductively as follows: Let $R_{0}=\sum_{i<\lambda_{1}} P_{i}$ where $\lambda_{1}$ is the least ordinal such that $\left|\sum_{i<\lambda_{1}} P_{i}\right| \geqq \sigma$. Assuming $R_{\alpha}$ has been defined for all $\alpha<\beta$, define $R_{\beta}=\sum_{\lambda_{\beta} \leqq i<\lambda_{\beta+1}} P_{i}$ where $\lambda_{\beta}$ is the least element of $\sigma$ such that $P_{\lambda_{\beta}} \cap R_{\alpha}=0$ for all $\alpha<\beta$, and $\lambda_{\beta+1}$ is the least element of $\sigma+1$ such that $\left|\sum_{\lambda_{\beta} \leq i<\lambda_{\beta+1}} P_{i}\right| \geqq\left|R_{\alpha}\right|$ for all $\alpha<\beta$. 
If $\lambda_{\beta}$ does not exist set $R_{\beta}=0$. It will be seen below that if $\lambda_{\beta}$ exists, $\lambda_{\beta+1}$ exists.

Note that $R_{\sigma}=0$ since $\sigma$ is an initial ordinal such that if $\beta<\sigma, \beta$ is not cofinal with $\sigma$ (i.e., $\sigma$ is regular). Note that $\sum_{i<\sigma} P_{i}=\sum_{i<\sigma} R_{i}$. If not, let $\eta$ be the least element of $\sigma+1$ such that $R_{\eta}=0$. If $\lambda_{\eta}=\sigma$ then $\sum_{i<\sigma} P_{i}=\sum_{i<\eta} R_{i}$. If $\lambda_{\eta}<\sigma$, then $\left|\sum_{\lambda_{\eta} \leqq i<\sigma} P_{i}\right|=\left|\sum_{i<\sigma} P_{i}\right| \geqq\left|R_{\alpha}\right|$ for all $\alpha<\eta$. Hence $R_{\eta} \neq 0$, a contradiction. Hence $\sum_{i<\sigma} R_{i}=\sum_{i<\sigma} P_{i}$.

Let $\eta$ be the least element of $\sigma+1$ such that $R_{\eta}=0$. Suppose $\eta$ is not a limit ordinal. Let $\eta=\gamma+1$. Then $R_{\gamma}=\sum_{\lambda_{\gamma} \leqq i<\sigma} P_{i}$ and $\left|R_{\gamma}\right| \geqq\left|R_{\alpha}\right|$ for all $\alpha<\gamma$. Construct a family $\left\{R_{\gamma}^{i}\right\}_{i \leqq \sigma}$ as above replacing 0 by $\lambda_{i}$. Let $\eta_{1}$ be the least ordinal such that $R_{r}^{\eta_{1}}=0$. Suppose $\eta_{1}$ is not a limit ordinal. Let $\eta_{1}=\gamma_{1}+1$. Then $\left|R_{r}^{r_{1}}\right|>\left|R_{r}^{\alpha}\right|$ for all $\alpha<\gamma_{1}$. In fact, $\left|R_{\gamma}^{\gamma_{1}}\right|>\sup _{\alpha<r_{1}}\left|R_{\gamma}^{\gamma}\right|$ since $\left|R_{\gamma}^{r_{1}}\right|=\left|R_{r}\right|$ and assuming otherwise we would have $R_{\gamma}=\left(\sum_{\alpha<\gamma_{1}} R_{\gamma}^{\alpha}\right) \oplus R_{\gamma}^{\gamma_{1}}$ with $\left|\sum_{\alpha<\gamma_{1}} R_{\gamma}^{\alpha}\right|=\left|R_{\gamma}\right|$ contradicting the construction of $R_{\gamma}$. Hence there exists $i, \lambda_{r_{1}} \leqq i<\sigma$ such that $\left|P_{i}\right| \geqq \sup _{\alpha<\gamma_{1}}\left|R_{r}^{\alpha}\right|$. This contradicts the construction of $R_{\gamma}^{\gamma_{1}}$. Hence $\eta_{1}$ is a limit ordinal.

Hence in either case ( $\eta$ or $\eta_{1}$ a limit ordinal) there exists a family of subsocles $\left\{R_{i}\right\}_{i<\eta}$ of $G[p]$ such that $\left|\sum_{i<\eta} R_{i}\right|=\left|\sum_{i<\sigma} P_{i}\right|$ and satisfying the conditions of Lemma 4. Thus there exists a subsocle $S$ of $\sum_{i<\eta} R_{i}$ satisfying conditions (a) and (b) of Lemma 4. Now $\sum_{i<\eta} R_{i}$ may not be all of $\sum_{i<\sigma}$ (the original $P_{i}$ ) and so we let $Q^{\prime}$ be such that $\sum_{i<\sigma}$ (the original $P_{i}$ ) $=\sum_{i<\eta} R_{i} \oplus Q^{\prime}$. We then have

$$
G[p]=\left\{\sum_{i<\eta} R_{i} \oplus Q^{\prime},\left(p^{\lambda} G\right)[p]\right\}=\left\{S \oplus Q^{\prime}, p^{\lambda} G[p]\right\}
$$

for each $\lambda<\tau$. Further,

$$
\left|G[p] / S \oplus Q^{\prime}\right| \geqq\left|\sum_{i<\eta} R_{i} / S\right| \geqq\left|\sum_{i<\eta} R_{i}\right|=|M|=s_{\tau}(G) .
$$

Hence $S \oplus Q^{\prime}$ supports a $p^{\tau}$-pure subgroup $H$ of $G$ such that $|G / H| \geqq$ $s_{\tau}(G)$. Thus $r_{\tau}(G) \geqq s_{\tau}(G)$. An application of this theorem appears in [1].

\section{REFERENCES}

1. D. O. Cutler and P. F. Dubois, A generalization of final rank of primary abelian groups, Canadian J. Math., vol. XXII, No. 6, (1970), 1118-1122.

2. L. Fuchs, Abelian Groups, Budapest 1958.

3. J. Irwin and E. A. Walker, On $N$-high subgroups of Abelian groups, Pacific J. Math., 11 (1961).

4. I. Kaplansky, Infinite Abelian Groups, University of Michigan Press, Ann Arbor, 1954.

5. R. J. Nunke, Homology and direct sums of countable Abelian groups, Math. Zeitschr. (101), (1967) 182-212.

6. J. E. Rubin, Set Theory for the Mathematician, Holden-Day, San Francisco 1967.

Received January 20, 1970.

University of CALIFornia, DAvis 



\section{PACIFIC JOURNAL OF MATHEMATICS}

\section{EDITORS}

\author{
H. SAMElson \\ Stanford University \\ Stanford, California 94305 \\ C. R. HOBBY \\ University of Washington \\ Seattle, Washington 98105
}

J. DugundjI

Department of Mathematics University of Southern California Los Angeles, California 90007

RICHARD ARENS

University of California

Los Angeles, California 90024

\section{ASSOCIATE EDITORS}
E. F. BECKENBACH
B. H. NeUmanN
F. WOLE
K. YOSHIDA

\section{SUPPORTING INSTITUTIONS}

\author{
UNIVERSITY OF BRITISH COLUMBIA \\ CALIFORNIA INSTITUTE OF TECHNOLOGY \\ UNIVERSITY OF CALIFORNIA \\ MONTANA STATE UNIVERSITY \\ UNIVERSITY OF NEVADA \\ NEW MEXICO STATE UNIVERSITY \\ OREGON STATE UNIVERSITY \\ UNIVERSITY OF OREGON \\ OSAKA UNIVERSITY \\ UNIVERSITY OF SOUTHERN CALIFORNIA
}

\author{
STANFORD UNIVERSITY \\ UNIVERSITY OF TOKYO \\ UNIVERSITY OF UTAH \\ WASHINGTON STATE UNIVERSITY \\ UNIVERSITY OF WASHINGTON \\ * * * \\ AMERICAN MATHEMATICAL SOCIETY \\ CHEVRON RESEARCH CORPORATION \\ NAVAL WEAPONS CENTER
}

The Supporting Institutions listed above contribute to the cost of publication of this Journal, but they are not owners or publishers and have no responsibility for its content or policies.

Mathematical papers intended for publication in the Pacific Journal of Mathematics should be in typed form or offset-reproduced, (not dittoed), double spaced with large margins. Underline Greek letters in red, German in green, and script in blue. The first paragraph or two must be capable of being used separately as a synopsis of the entire paper. The editorial "we" must not be used in the synopsis, and items of the bibliography should not be cited there unless absolutely necessary, in which case they must be identified by author and Journal, rather than by item number. Manuscripts, in duplicate if possible, may be sent to any one of the four editors. Please classify according to the scheme of Math. Rev. Index to Vol. 39. All other communications to the editors should be addressed to the managing editor, Richard Arens, University of California, Los Angeles, California, 90024.

50 reprints are provided free for each article; additional copies may be obtained at cost in multiples of 50 .

The Pacific Journal of Mathematics is published monthly. Effective with Volume 16 the price per volume (3 numbers) is $\$ 8.00$; single issues, $\$ 3.00$. Special price for current issues to individual faculty members of supporting institutions and to individual members of the American Mathematical Society: $\$ 4.00$ per volume; single issues $\$ 1.50$. Back numbers are available.

Subscriptions, orders for back numbers, and changes of address should be sent to Pacific Journal of Mathematics, 103 Highland Boulevard, Berkeley, California, 94708.

PUBLISHED BY PACIFIC JOURNAL OF MATHEMATICS, A NON-PROFIT CORPORATION

Printed at Kokusai Bunken Insatsusha (International Academic Printing Co., Ltd.), 7-17, Fujimi 2-chome, Chiyoda-ku, Tokyo, Japan. 


\section{Pacific Journal of Mathematics}

\section{Vol. 37, No. $2 \quad$ February, 1971}

Charles Compton Alexander, Semi-developable spaces and quotient images of metric spaces .................................... 277

Ram Prakash Bambah and Alan C. Woods, On a problem of Danzer. . . . . . . . . 295

John A. Beekman and Ralph A. Kallman, Gaussian Markov expectations and related integral equations . ....................................

Frank Michael Cholewinski and Deborah Tepper Haimo, Inversion of the Hankel

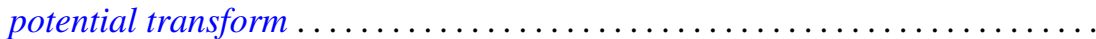

John H. E. Cohn, The diophantine equation

$$
Y(Y+1)(Y+2)(Y+3)=2 X(X+1)(X+2)(X+3) \ldots \ldots \ldots \ldots \ldots
$$

Philip C. Curtis, Jr. and Henrik Stetkaer, A factorization theorem for analytic

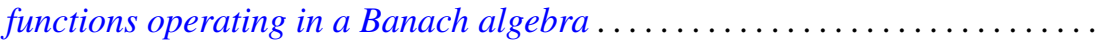

Doyle Otis Cutler and Paul F. Dubois, Generalized final rank for arbitrary limit ordinals

Keith A. Ekblaw, The functions of bounded index as a subspace of a space of entire functions

Dennis Michael Girard, The asymptotic behavior of norms of powers of

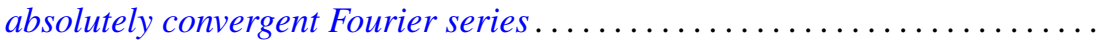

John Gregory, An approximation theory for elliptic quadratic forms on Hilbert spaces: Application to the eigenvalue problem for compact quadratic forms. 383

Paul C. Kainen, Universal coefficient theorems for generalized homology and stable cohomotopy.

Aldo Joram Lazar and James Ronald Retherford, Nuclear spaces, Schauder bases, and Choquet simplexes.

David Lowell Lovelady, Algebraic structure for a set of nonlinear integral operations

John McDonald, Compact convex sets with the equal support property . 429

Forrest Miller, Quasivector topologies

Marion Edward Moore and Arthur Steger, Some results on completability in commutative rings.

A. P. Morse, Taylor's theorem

Richard E. Phillips, Derek J. S. Robinson and James Edward Roseblade, Maximal subgroups and chief factors of certain generalized soluble groups.

Doron Ravdin, On extensions of homeomorphisms to homeomorphisms ...

John William Rosenthal, Relations not determining the structure of $\mathrm{L}$

Prem Lal Sharma, Proximity bases and subbases ........... .

Larry Smith, On ideals in $\Omega_{*}^{u}$. .

Warren R. Wogen, von Neumann algebras generated by operators similar to normal operators

R. Grant Woods, Co-absolutes of remainders of Stone-Čech 